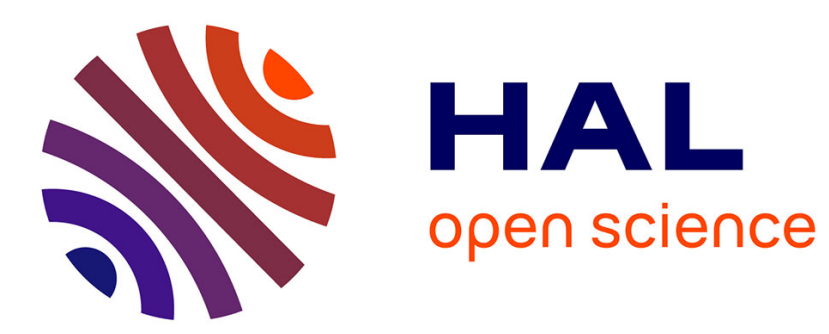

\title{
A principal component analysis of the relationship between the external body shape and internal skeleton for the upper body
}

\author{
Agathe Nerot, Wafa Skalli, Xuguang Wang
}

\section{- To cite this version:}

Agathe Nerot, Wafa Skalli, Xuguang Wang. A principal component analysis of the relationship between the external body shape and internal skeleton for the upper body. Journal of Biomechanics, 2016, 49 (14), pp.3415-3422. 10.1016/j.jbiomech.2016.09.006 . hal-01465073

\section{HAL Id: hal-01465073 \\ https://hal.science/hal-01465073}

Submitted on 10 Feb 2020

HAL is a multi-disciplinary open access archive for the deposit and dissemination of scientific research documents, whether they are published or not. The documents may come from teaching and research institutions in France or abroad, or from public or private research centers.
L'archive ouverte pluridisciplinaire HAL, est destinée au dépôt et à la diffusion de documents scientifiques de niveau recherche, publiés ou non, émanant des établissements d'enseignement et de recherche français ou étrangers, des laboratoires publics ou privés. 


\title{
A principal component analysis of the relationship between the external body shape and internal skeleton for the upper body
}

\author{
A. Nerot ${ }^{\mathrm{a}, \mathrm{b}}, \mathrm{W}$. Skalli ${ }^{\mathrm{b}}, \mathrm{X}$. Wang ${ }^{\mathrm{a}, *}$ \\ a Univ Lyon, Université Claude Bernard Lyon 1, IFSTTAR, UMR_T9406, LBMC, F69622 Lyon, France \\ ${ }^{\mathrm{b}}$ Arts et Métiers ParisTech, LBM/institute de Biomécanique humaine Georges Charpak, 151 Boulevard de l'hôpital, 75013 Paris, France
}

Keywords:

Internal points prediction

PCA

Biplanar X-rays

3D reconstruction

Subject-specific human model

\begin{abstract}
A B S T R A C T
Recent progress in 3D scanning technologies allows easy access to 3D human body envelope. To create personalized human models with an articulated linkage for realistic re-posturing and motion analyses, an accurate estimation of internal skeleton points, including joint centers, from the external envelope is required. For this research project, 3D reconstructions of both internal skeleton and external envelope from low dose biplanar X-rays of 40 male adults were obtained. Using principal component analysis technique (PCA), a low-dimensional dataset was used to predict internal points of the upper body from the trunk envelope. A least squares method was used to find PC scores that fit the PCA-based model to the envelope of a new subject. To validate the proposed approach, estimated internal points were evaluated using a leave-one-out (LOO) procedure, i.e. successively considering each individual from our dataset as an extra-subject. In addition, different methods were proposed to reduce the variability in data and improve the performance of the PCA-based prediction. The best method was considered as the one providing the smallest errors between estimated and reference internal points with an average error of $8.3 \mathrm{~mm}$ anterior-posteriorly, $6.7 \mathrm{~mm}$ laterally and $6.5 \mathrm{~mm}$ vertically.

As the proposed approach relies on few or no bony landmarks, it could be easily applicable and generalizable to surface scans from any devices. Combined with automatic body scanning techniques, this study could potentially constitute a new step towards automatic generation of external/internal subject-specific manikins.
\end{abstract}

\section{Introduction}

Subject-specific models of the human body are required for motion analysis in many fields such as in ergonomics and clinical applications (Chaffin, 2005; Blanchonnette, 2010; Regazzoni et al., 2015). Both the representation of the external body shape and the internal skeleton provide required information to fully understand the interaction of an individual with his/her environment and its impacts on the musculo-skeletal system (e.g. interaction with workplace, medical device...). Recent progress in 3D body scanning permitted the development of statistical models of the external body shape in order to create custom avatars from an incomplete or noisy 3D scan dataset (Allen and Curless, 2003; Park and Reed, 2014; Park et al., 2015). However, estimating the location of internal joint centers from the external body envelope still remains a challenging issue.

\footnotetext{
* Correspondence to: LBMC - Laboratoire de Biomécanique et Mécanique des Chocs (UMR-T 9406 IFSTTAR/UCBL), IFSTTAR - Institut Français des Sciences et Technologies des Transports, de l'Aménagement et des Réseaux 25, avenue François Mitterrand, Case 24, F-69675 Bron, France.

E-mail address: xuguang.wang@ifsttar.fr (X. Wang).
}

For this purpose, statistical body shape models, including joint centers coordinates, have recently been introduced for different applications. In clinical monitoring, prediction of the scoliotic spinal curvature from back shape surface was performed using principal components analysis (PCA) on a database of back 3D surfaces and spine radiographs (Huysmans et al., 2005; Bergeron et al., 2005). In computergraphics, a statistical model of body shape and pose including an 18segment skeleton was used to create articulated avatars from body scanner (Anguelov et al., 2005) or Microsoft Kinect acquisitions (Weiss et al., 2011). It should be noted that joint centers used for modifying avatars' shape and pose are not necessarily anatomically correct. In an attempt to improve the realism of the internal skeleton for biomechanical analysis, Park and Reed (2015) and Reed et al. (2014) introduced a statistical model built from a database including 3D scans and estimated joint centers from palpated bony landmarks. As an alternative, Reed et al. (2015) proposed a statistical body shape model of the body envelope and palpated bony landmarks location. Using the PCA-based matching of 3D scans, the idea was to predict bony landmarks at first and then to use them for locating joint centers from regressions (Reed et al., 1999). However estimation of joint centers based on manual bony landmarks palpation is time consuming, prone to incertitude (Harlick et al., 2007) and results may be operator dependent (Croce et al., 1999). 
Using a PCA-based method for estimating internal joint center by fitting a statistical body shape model to exterior surface is not novel. However there is no study which has quantified the accuracy of this method on an asymptomatic population due to the lack of data containing both external body shape and internal skeleton.

Biplanar X-rays methods (Dubousset et al., 2010) have been developed to reconstruct both the bones and the body envelope in three dimensional space (3D) from a standing posture based on two radiographic views (Mitton et al., 2006; Humbert et al., 2009; Quijano et al., 2013; Aubert et al., 2014; Nérot et al., 2015a). Using this technology the aim of this study was to explore whether a PCA-based model could be applied for accurately predicting internal skeleton points, such as joint centers or other bony landmarks, from the external body shape as input. A focus was given on the upper body region for predicting spine and hip joint centers, along with some pelvic landmarks. This region represents an important part of the kinematic chain used for posture and motion analysis and remains challenging to locate internal points from external data.

\section{Materials and methods}

\subsection{Data}

After receiving approval from the ethics committee (CPP 06036) and written consent from the participants, the biplanar X-rays of 40 male subjects $\left(17.7<\mathrm{BMI}<33.2 \mathrm{~kg} / \mathrm{m}^{2}\right.$, $1.65<$ height $<1.88 \mathrm{~m}, 60.0<$ weight $<103.3 \mathrm{~kg}, 20<$ age $<75$ years) were obtained with an EOS system (EOS Imaging, Paris, France). While standing in the EOS cabin, participants were asked to adopt a free standing position with elevated arms as described in Faro et al. (2004) and with slightly shifted feet (Chaibi et al., 2012). 3D reconstructions of body envelope and skeleton (including pelvis and spine) were performed from the participants' biplanar X-rays (Mitton et al., 2006, Humbert et al., 2009). The envelope reconstruction method already described in our previous work (Nérot et al., 2015b), consists of deforming a template of the human body envelope to match the visible skin contours on the X-rays (Fig. 1a). Each reconstructed envelope has the same topology with orderly distributed vertices. The trunk region was segmented once and for all on the template between the neck, arms and groins with 1689 vertices and 3374 triangular polygons (Fig. 1b). By identifying the mesh components belonging to the predefined region, the trunk was automatically extracted from the reconstructed body envelopes. From the pelvis and spine 3D reconstructions, twenty-six internal skeleton points were automatically extracted (Fig. 1c), including C7/T1 to L5/S1, right and left hip joint centers
$(\mathrm{HJC})$, right and left anterior-superior iliac spine (ASIS), right and left posterior-superior iliac spine (PSIS), right and left insertions of the semitendineous muscles on the ischial tuberosities of the pelvis (IT). The intervertebral joint centers were defined as the middle points of the segments joining the barycenters of the upper vertebrae under plates and the lower vertebrae upper plates (Humbert et al., 2009). The hip joint center corresponded to the femoral head center calculated as in Chaibi et al. (2012).

\subsection{PCA analysis}

Trunk external shape meshes and internal skeleton points were aligned in a global coordinate system (GCS) centered at the origin of the pelvis reference frame (Table 1). The ASIS and PSIS landmarks were virtually palpated on the reconstructed pelvis. The closest point of the ASIS and PSIS on the envelope mesh was considered as an estimation of the regular palpated landmarks on the skin (Fig. 1d).

PCA was used (Jolliffe, 2002) to reduce the dimensionality of the dataset. The 3D coordinates of the 1689 trunk mesh vertices from the 40 subjects were gathered in a matrix $\boldsymbol{\Psi}_{n * p}$, with $n$ corresponding to 40 subjects (observations) and $p$ to $3^{*} 1689$ vertex coordinates (variables). The $q(=3 * 26)$ coordinates of the 26 internal points were appended to $\boldsymbol{\Psi}_{n * p}$ resulting in a matrix $\boldsymbol{\Psi}_{n *(p+q)}$. A smaller set of ordered variables called principal component (PC) scores, was obtained with PCA, so that the first PCs retained most of the variation in the original dataset. PC scores were calculated for each subject. An intuitive interpretation of the PCs was performed by varying the scores along each component from (mean $-2 \mathrm{SD}$ ) to (mean +2SD).

\subsection{Prediction of internal skeleton points}

From the PCA of data, assume that $M$ main PCs $\mathbf{u}_{\mathrm{j}}(j=1, M)$ are retained. The estimated $p$ vertex coordinates of the body shape without internal skeleton points of an extra subject outside the sample data $\boldsymbol{\Psi}_{e}$ can be expressed approximately:

$\boldsymbol{\Psi}_{e}(1: p) \approx \overline{\boldsymbol{\Psi}}(1: p)+\sum_{j=1}^{M} c_{j} \boldsymbol{u}_{j}(1: p)$

where $\overline{\boldsymbol{\Psi}}$ is the average from the sample data sets and $c_{j}$ is the unknown score associated with $j^{\text {th }}$ PC. $c_{j}$ can be estimated by the mean least squares method which minimizes the weighted summed square of residuals $(r)$ between the coordinates of the estimated $\boldsymbol{\Psi}_{e}$ and target $\boldsymbol{\Psi}_{t}$ envelope vertices:

$\operatorname{minimize} f\left(c_{1}, c_{2}, \ldots c_{M}\right)=\sum_{i=1}^{p} w_{i} r_{i}^{2}=\boldsymbol{r} W \boldsymbol{r}^{T}$

where $\boldsymbol{r}=\boldsymbol{\Psi}_{e}-\boldsymbol{\Psi}_{t}=\overline{\boldsymbol{\Psi}}+\sum_{j=1}^{M} c_{j} \boldsymbol{u}_{j}-\boldsymbol{\Psi}_{t}$ and $\mathrm{W}$ the diagonal weighting matrix. a

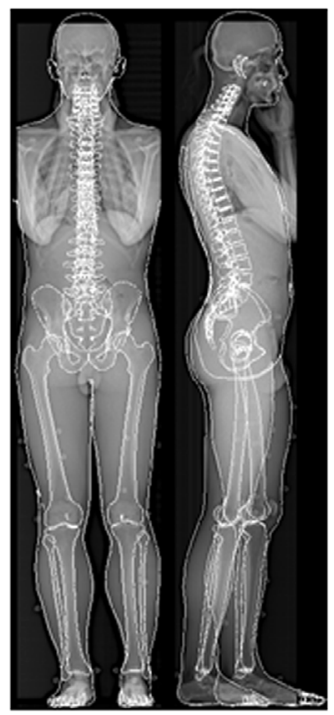

b

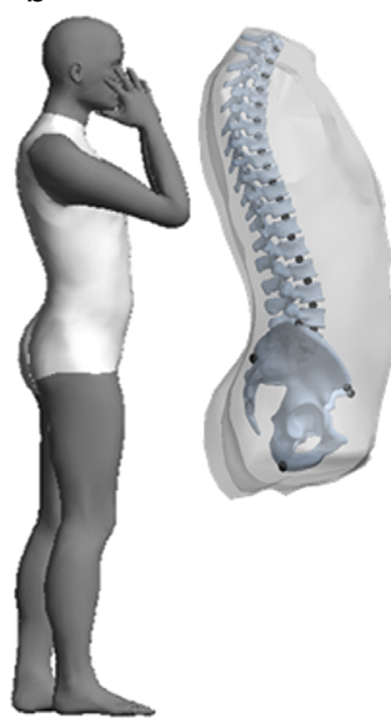

d

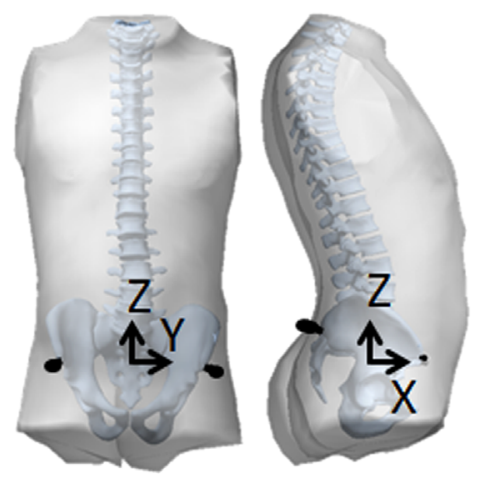

e

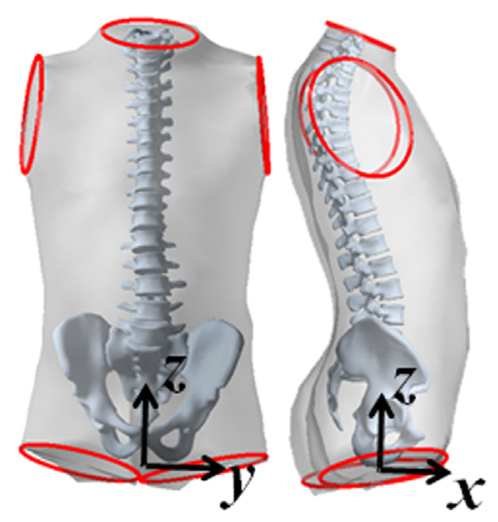

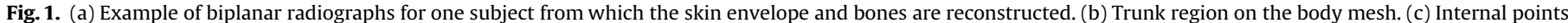

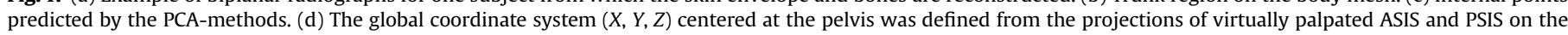

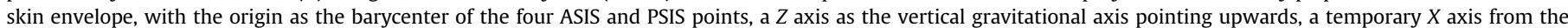

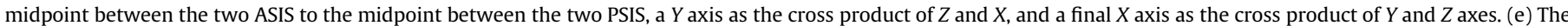

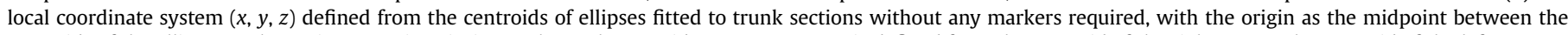

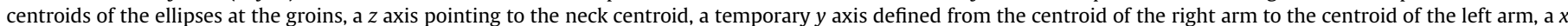
axis as the cross product of $z$ and $y$ and a final $y$ axis as the cross product of $x$ and $z$ axes. 
Table 1

GCS definition.

\begin{tabular}{ll}
\hline Origin & Barycenter of the ASIS and PSIS landmarks \\
\hline$Z$ & Vertical gravitational axis pointing upwards \\
$X$ & A temporary $X$ axis was defined from the midpoint between the two \\
& ASIS and the midpoint between the two PSIS, pointing anteriorly. The \\
& final $X$ axis was the cross product of $Y$ and $Z$ axes \\
$Y$ & $Y$ axis was calculated as the cross product of $Z$ and temporary $X$ axes \\
\hline
\end{tabular}

The $q$ coordinates of the internal skeletal points can then be estimated once the PC scores $c_{j}$ are known

$\boldsymbol{\Psi}_{e}(p+1: p+q) \approx \overline{\boldsymbol{\Psi}}(p+1: p+q)+\sum_{j=1}^{M} c_{j} \boldsymbol{u}_{j}(p+1: p+q)$

In this study, the number of $M$ main PCs was chosen so that the cumulative sum of the variance explained by the $M$ first principal components equals $99 \%$ of the data variance.

A matching error was defined as the mean distance between the PCA-based and target envelopes. The matching error reflected the ability of a PCA model to describe the trunk envelope of an extra individual.

\subsubsection{Evaluation of the proposed approach}

To evaluate the proposed approach for predicting internal skeleton points, a leaveone-out (LOO) procedure was performed using the data from the 40 males in the present study. The PCA model was first built from $n-1$ subjects, then the internal points of the $n^{\text {th }}$ extra subject were predicted only using his skin envelope. The residual between estimated internal points and anatomical reference points was calculated. This procedure was iteratively repeated until each subject had been considered as an extra subject once. The standard error of estimate (SEE) over the 40 residuals was considered to estimate the accuracy of predicted location of the internal points.

\subsubsection{Different methods for predicting internal points}

Two different strategies were tested to reduce data variability. The first strategy was to align the trunk meshes using a local coordinate system (LCS). The second strategy was to normalize the data by trunk anthropometric dimensions. Moreover, as internal points may be more related to some external points than others, a weighting strategy was also tested.

2.3.2.1. Global and local coordinate systems. The coordinates of trunk envelope vertices and internal points were expressed either in the GCS centered at the pelvis or in a LCS (Fig. 1e).

For the LCS, five ellipses were fitted using the method of least squares to the five trunk extremities, i.e. neck, arms and groins (Table 2).

2.3.2.2. Vertices weighting ( $V W$ ). To favor the matching of the external points which are close to sub-cutaneous bone reliefs, a higher weight coefficient was allocated to these points. The selected surface points for a higher weighting are shown in Fig. 2. They were the points of the external posterior profil close to the vertebral spinous processes reliefs and the upper parts of the iliac crests for the pelvis. The vertices forming a pelvis belt passing through the apex of the lumbar lordosis to the most anterior point of the trunk silhouette approximating ASIS height were allocated also with a higher weight coefficient in order to preserve pelvis depth and width. The mean ASIS height from the sample data, estimated as $28 \%$ of the distance between the middle of the arms sections and the origin (Fig. 2), was used. In addition, the vertices at the upper and lower trunk extremities, and arms sections were weighted to preserve trunk height and width.

2.3.2.3. Coordinates normalization. To reduce the variability due to the change of anthropometric dimensions, the coordinates were normalized by trunk height (TN). Trunk height was defined from the centroid of the neck ellipse to the origin.

By combining the ways of reducing data variability and weighting strategy, four different methods were proposed and tested in the present work (Table 3):

Table 2

LCS definition.

Origin Midpoint between the centroids of the ellipses at the groins

$z \quad$ Axis pointing to the neck centroid

$y \quad$ A temporary $y$ axis was defined from the centroid of the right arm to the centroid of the left arm. The final $y$ axis was the cross product of $z$ and $x$ axes.

$x \quad x$ axis was calculated as the cross product of $z$ and temporary $y$ axes

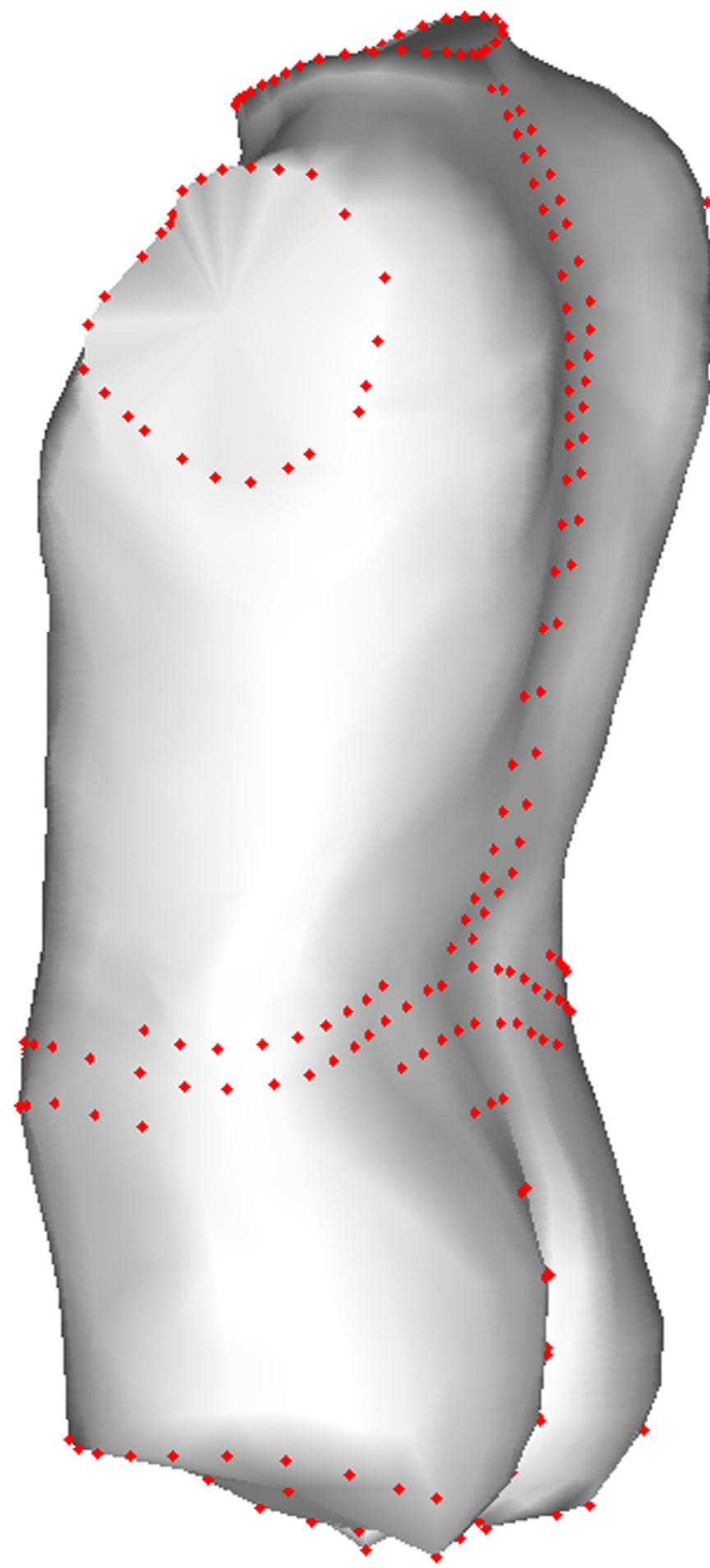

Fig. 2. Mesh vertices selected for a higher weighting in the objective function.

\section{Results}

\subsection{Principal components analysis}

PCA was performed on the raw data expressed in a GCS (Method 1). 27 first PCs accounted for $99 \%$ of the variance in data (Fig. 3b). Fig. 3a shows the trunk shape variation with the internal skeleton points of interest along the first five PCs which account for nearly $80 \%$ of variance. Visually, these variances could be explained by trunk height (PC1, PC2, PC3), waist circumference (PC1, PC4) trunk depth 
Table 3

PCA-based methods.

$\begin{array}{ll}\text { Method 1 } & \text { Basic configuration (GCS) } \\ \text { Method 2 } & \text { GCS }+ \text { VW (weighting) } \\ \text { Method 3 } & \text { GCS }+ \text { TN (normalizing by trunk height) } \\ \text { Method 4 } & \text { LCS (local coordinate system) }\end{array}$

Note that Method 4 is anatomical landmark independent.
(PC2) as well trunk orientation in space (PC1 for posterior-anterior rotation, PC2 for left axial rotation, PC3 for right axial rotation, PC5 for lateral rotation). PC1 and PC4 could be explained by spine curvature (kyphosis and lordosis). In addition, thoracic joint centers location was directly affected by external shape variation such as trunk height (PC1, PC2, PC3), spine curvature (PC1, PC4), and rotations (PC1, PC2, PC3, PC5), while lumbar joints did not seem affected by variation in

a
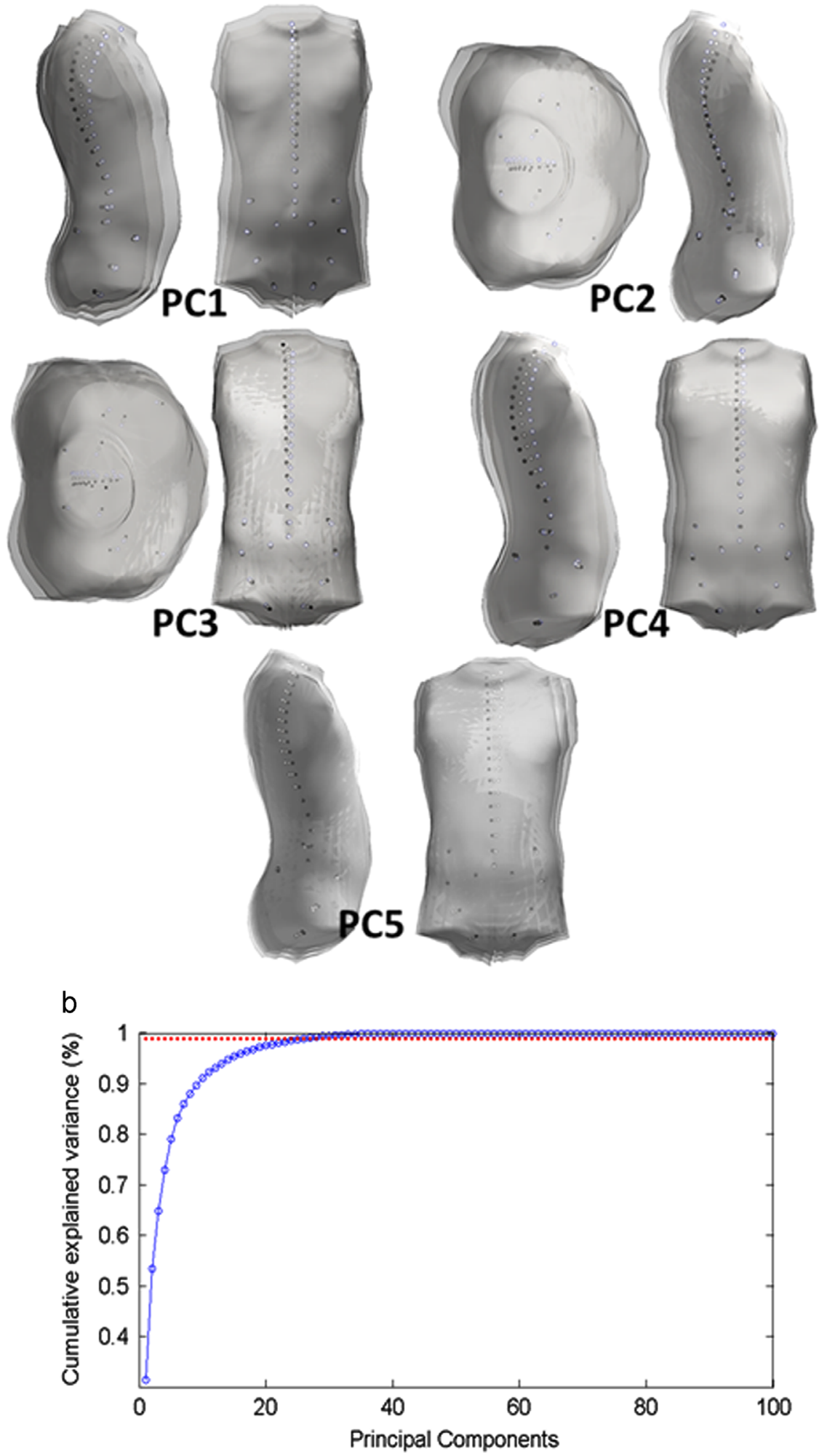

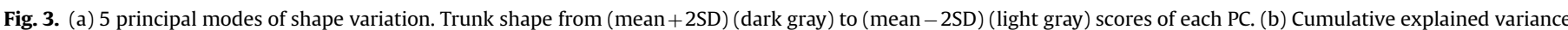
as a function of the number of principal components. 
belly depth. Pelvis landmarks location was affected by pelvis depth and width (PC1, PC4), and trunk height (PC3).

Based on this analysis, different strategies were proposed to reduce the variance in data. Variation in trunk orientation in space reflected different postures adopted by participants while standing and justified the use of a LCS. Normalization by trunk height was proposed to reduce the variability in body size. In addition, due to the proximity between the spinous processes of the internal spinal vertebra and the back surface, different vertices weighting coefficients were tested in order to enforce the matching of the dorsal profile.

\subsection{Surface fitting and location of internal skeleton points}

Surface matching errors and standard errors of estimate (SEE) of a set of selected internal points are presented in Table 4. Here are the main observations when comparing the four proposed methods in Table 4:

- The surface matching errors were quite similar among the different methods and remained below $10 \mathrm{~mm}$ on average.

- The residual distances of all internal points ranged from 12.5 (Method 1) to $14.7 \mathrm{~mm}$ (Method 4) on average.

- Weighting of the selected surface points had almost no effect on the location of estimated internal points.

- Data normalization by trunk height had no effect on reduction of data dimensionality.

- The use of the LCS decreased the number of PCs required to explain 99\% of the variance, from 27 (Method 1) to 23 (Method 4). However, bigger errors of the internal points were observed when using the LCS, especially for pelvic points.

- When comparing different internal points, the highest error was observed for L2/L3 for all four methods.

\section{Discussion}

This study was motivated by the need of estimating internal skeleton points from skin envelope for posture and motion analysis. A PCA was used for analyzing the relationship between the external body shape and internal skeleton for the upper body. While fitting a PCA-based statistical body shape model to exterior 3D surfaces for predicting internal points is not novel, the original contribution of this study was to build the model from subject-specific radiographs in order to improve and quantify accuracy of prediction. Four different PCA-based methods were tested for locating internal points. The lowest error of the internal points obtained with Method 1 (basic configuration) was $8.3 \mathrm{~mm}$ in $x, 6.7 \mathrm{~mm}$ in $y$ and $6.5 \mathrm{~mm}$ in $z$ directions on average. Method 1 does not require any tuning parameters compared to Methods 2-4. The all four PCA matching methods provided higher accuracy for predicting hip and vertebral joints than existing digital manikins such as RAMSIS (Human Solutions) and

Table 5

Pearson correlation coefficients between the errors of the internal points in anterior-posterior direction and surface matching errors, body height, age, T4-T12 kyphosis and L1-L5 lordosis angles. Correlations with a p-Value less than 0.05 are marked with *. T4-T12 kyphosis angle and L1-L5 lordosis angles were calculated using the endplates method as described in Schwab et al. (2006).

\begin{tabular}{lllllll}
\hline & Surface matching error & Height & Weight & Age & T4-T12 & L1-L5 \\
\hline HipR & 0.3 & 0.1 & 0 & 0 & 0 & 0.2 \\
HipL & 0.2 & 0 & 0 & 0 & 0.1 & 0.1 \\
ASISR & -0.1 & -0.1 & 0 & -0.2 & -0.2 & 0.1 \\
ASISL & 0.1 & 0 & 0 & -0.1 & -0.2 & 0.1 \\
ITR & 0.2 & 0.1 & 0 & -0.1 & 0.1 & 0.2 \\
ITL & 0.3 & 0.1 & 0 & -0.1 & 0.2 & 0.2 \\
PSISR & 0.3 & 0.2 & 0.1 & -0.1 & -0.1 & 0.2 \\
PSISL & 0.1 & 0.3 & 0.1 & -0.1 & -0.1 & 0.2 \\
T1/T2 & $0.3 *$ & 0 & 0 & -0.1 & -0.1 & 0.2 \\
T4/T5 & 0.1 & 0.1 & 0.1 & 0 & 0.1 & 0.2 \\
T5/T6 & 0.1 & 0.1 & 0.1 & 0 & 0.2 & 0.2 \\
T6/T7 & 0.1 & 0.1 & 0.1 & 0 & 0.2 & 0.2 \\
T7/T8 & 0.1 & 0.1 & 0.1 & 0 & 0.3 & 0.2 \\
T8/T9 & 0.1 & 0.1 & 0 & 0 & $0.4^{*}$ & 0.2 \\
T9/T10 & 0.1 & 0 & 0 & 0.1 & $0.4^{*}$ & 0.1 \\
T10/T11 & 0.1 & 0 & -0.1 & 0.2 & $0.4^{*}$ & 0.1 \\
T11/T12 & 0.1 & -0.1 & -0.1 & 0.3 & $0.4^{*}$ & -0.1 \\
T12/L1 & 0.1 & -0.2 & -0.2 & 0.3 & 0.3 & -0.3 \\
L1/L2 & 0.1 & -0.2 & -0.2 & 0.3 & 0.1 & $-0.4^{*}$ \\
L2/L3 & 0 & -0.2 & -0.2 & 0.3 & 0.1 & $-0.5^{*}$ \\
L3/L4 & 0 & -0.1 & -0.2 & 0.3 & 0 & $-0.5^{*}$ \\
L4/L5 & 0 & 0 & -0.1 & 0.2 & -0.1 & -0.2 \\
L5/S1 & 0 & 0.1 & 0 & 0 & -0.2 & 0.2 \\
\hline
\end{tabular}

HipR/HipL: right/left hip joint center; ASISR/ASISL: right/left anterior-superior iliac crest; ITR/ITL: right/left IT; PSISR/PSISL: right/left posterior-superior iliac crest.'

$$
{ }^{*} p<0.05 \text {. }
$$

Table 4

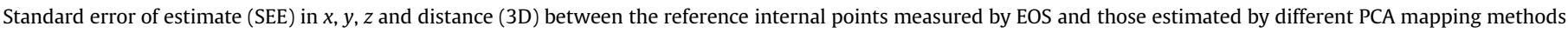

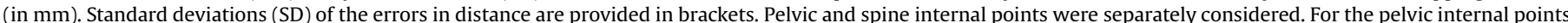

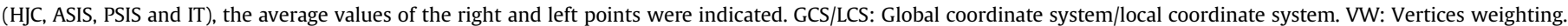
$\mathrm{TN}$ : normalization by trunk height.

\begin{tabular}{|c|c|c|c|c|c|c|c|c|c|c|c|c|c|c|c|}
\hline \multirow[t]{2}{*}{ Method } & \multirow[t]{2}{*}{$i$} & \multirow[t]{2}{*}{ PC } & & \multirow{2}{*}{$\begin{array}{l}\text { Surface } \\
\text { matching } \\
\text { error }\end{array}$} & \multicolumn{4}{|c|}{ Pelvic internal points: SEE (SD) } & \multicolumn{6}{|c|}{ Spine internal points: SEE (SD) } & \multirow[t]{2}{*}{ All $^{\mathrm{a}}$} \\
\hline & & & & & Hip & ASIS & PSIS & IT & T1/T2 & T4/T5 & T8/T9 & T12/L1 & L2/L3 & L5/S1 & \\
\hline \multirow[t]{4}{*}{ GCS } & 1 & 27 & $x$ & & 6.4 & 5.5 & 7 & 8.6 & 7.5 & 9.4 & 8.6 & 8.2 & 9.3 & 9.5 & $8.3(1.6)$ \\
\hline & & & $y$ & & 6.4 & 8.8 & 6.8 & 8.4 & 4.6 & 5.4 & 6.5 & 5.9 & 8.5 & 4.4 & $6.7(1.5)$ \\
\hline & & & $z$ & & 5.8 & 8.3 & 7.2 & 5.6 & 6.3 & 6 & 5.5 & 6.6 & 8 & 7.2 & $6.5(1.1)$ \\
\hline & & & 3D & 7.8 (1.9) & $10.8(4.5)$ & $13.2(5.5)$ & $12.2(5.2)$ & $13.3(6.2)$ & $10.8(4.3)$ & $12.4(5.7)$ & $12.1(4.5)$ & $12(4.5)$ & $14.9(6.0)$ & $12.7(5.2)$ & $12.6(1.5)$ \\
\hline \multirow[t]{4}{*}{$G C S+V W$} & 2 & 27 & $x$ & & 6.7 & 5.2 & 7 & 8.7 & 6.8 & 9.6 & 8.4 & 7.9 & 8.8 & 9.1 & $8.1(1.6)$ \\
\hline & & & $y$ & & 6.4 & 8.5 & 6.8 & 8.2 & 4.5 & 5.3 & 6.4 & 5.9 & 8.2 & 4.5 & $6.6(1.4)$ \\
\hline & & & $z$ & & 5.5 & 8.4 & 7.2 & 5.7 & 6.2 & 5.8 & 5.7 & 6.9 & 8.3 & 7.1 & $6.6(1.1)$ \\
\hline & & & 3D & $8.0(1.9)$ & $10.8(4.4)$ & $13(5.3)$ & $12.2(5.2)$ & $13.4(6.3)$ & $10.2(4.1)$ & $12.4(5.9)$ & $12(4.9)$ & $12(4.3)$ & $14.6(5.9)$ & $12.4(4.9)$ & $12.5(1.4)$ \\
\hline \multirow[t]{4}{*}{$G C S+T N$} & 3 & 27 & $\boldsymbol{x}$ & & 5.5 & 5.8 & 6.9 & 7.3 & 8.3 & 8.6 & 8.1 & 7.8 & 8.7 & 10 & $8,0(1.4)$ \\
\hline & & & $y$ & & 7.5 & 9.7 & 6.6 & 10 & 4.6 & 5.3 & 6.9 & 7 & 9.4 & 4.3 & $7.1(2,0)$ \\
\hline & & & $z$ & & 6.2 & 8.2 & 6.9 & 5.1 & 7.1 & 6.4 & 5.8 & 6.4 & 8 & 7.2 & $6.7(1.0)$ \\
\hline & & & 3D & $7.7(1,7)$ & $11,2(5,4)$ & $14,0(5.7)$ & $11,8(6,8)$ & $13,6(7,2)$ & $11,8(8,1)$ & $12,0(8,5)$ & $12,2(8,0)$ & $12,3(7,7)$ & $15,1(8,6)$ & $13,0(9,9)$ & $12.8(1.4)$ \\
\hline \multirow[t]{4}{*}{ LCS } & 4 & 23 & $x$ & & 9.4 & 10 & 8.8 & 9.4 & 7.1 & 9.4 & 7.7 & 7.1 & 7.8 & 9.5 & $8,8(2,0)$ \\
\hline & & & $y$ & & 7.4 & 10.7 & 10.6 & 8.5 & 4.8 & 5.1 & 8.1 & 6.5 & 7.2 & 6.2 & $7.5(2,0)$ \\
\hline & & & $z$ & & 7.1 & 13.2 & 10.6 & 7 & 6.9 & 6.6 & 6.4 & 7.9 & 8.9 & 8.9 & $8.1(1.9)$ \\
\hline & & & 3D & $7,4(1,7)$ & $14,0(5,8)$ & $19,7(7,4)$ & $17,4(5,8)$ & $14,6(5,8)$ & $11,0(3,9)$ & $12,5(5,6)$ & $12,9(4,3)$ & $12,4(4.2)$ & $13,9(5,3)$ & $14,4(5,7)$ & $14.3(2.8)$ \\
\hline
\end{tabular}

a All 27 internal points are included: all spine joints from T1/T2 to L5/S1 along with all pelvic landmarks considered in this study. 
Human Builder (Dassault Systèmes) (Nérot et al., 2015a). In addition the proposed approach requires few or no palpation of bony landmarks for the prediction of 26 internal points. It can be easily applied to a 3D scan surface thanks to an automatic segmentation process. This is a great advantage compared to currently existing regression methods based on the manual palpation of different bony landmarks for hip joint centers (Bell and Pedersen, 1989; Snyder et al., 1972; Seidel et al., 1995; Reed et al., 1999; Peng et al., 2015) and spine joint centers estimation (Sicard and Gagnon, 1993). In terms of prediction accuracy, a comparable magnitude of errors was found by the first three methods for L5/S1 and hip joint centers compared to the bony landmarks based regression methods reported in Peng et al. (2015) showing errors in distance of approximately $10 \mathrm{~mm}$. For the spinal joint centers, an average error of less than $15.5 \mathrm{~mm}$ was found for all four methods, lower than the error found in Sicard and Gagnon (1993) $(17.4 \pm 10.8 \mathrm{~mm})$, requiring the palpation of the spinous process of L5 and additional anthropometric measurements. It should be noted that the palpation of the spinous processes was highly operator dependent (Harlick et al., 2007).

The use of a LCS and normalization by trunk height (TN) aimed at reducing the variability in data, mainly due to the variation in anthropometric dimensions and in trunk orientation. As expected, the use of a LCS (Method 4) reduced the number of PCs representing 99\% of the variance, from 27 (Method 1) to 23 PCs. But data normalization by trunk height did not allow decreasing the number of main PCs. Therefore using a LCS provides an interesting approach for reducing the number of unknowns to be predicted, especially when the whole trunk surface is not available but only a reduced set of external inputs. The LCS proposed in the present work for aligning trunk surfaces did not require the palpation of any anatomical landmarks, but was dependent on the choice of segmentation. Higher errors were found for the pelvic points, especially for the ASIS and PSIS. In the GCS, the ASIS and PSIS were used to define the origin of the coordinate system. This suggests the need of palpation of some ALs for defining LCS in order to improve prediction of pelvic internal points.
The weighting strategy for favoring the matching of the vertebral spinous processes reliefs did not reduce the estimation error of internal points. Different weighting coefficients ranging from 5 to 30 were tested. Increasing the weighting coefficients slightly reduced the mean distance between weighted external points from 5.3 to $4.8 \mathrm{~mm}$ and slightly increased the mean distance for all surface points from 8.0 to $8.9 \mathrm{~mm}$ as expected. However it did not reduce the errors of prediction of the internal points ranging from $12.8 \mathrm{~mm}$ with a weighting of $5-13.0 \mathrm{~mm}$ with a weighting of 30 . Instead of taking the whole trunk into consideration, a different PCA model could be proposed only from the rear surface points of the torso. For instance, Huysmans et al. (2005) and Bergeron et al. (2005) only selected the back surface while Drerup and Hierholzer (1996) only selected the midline through the spinous processes on the back surface in their PCA-based models for predicting spinal lateral deviations. Considering only the rear part of the torso would allow reducing the variability in the data.

The critical issue for estimating internal skeleton points from the external body surface is that the characteristics of the internal skeleton may not be well related to those of external shape. For example, for an adult, body shape variation induced by weight change may not necessarily cause variation in the skeleton location. In other words, would a perfect matching of the external envelope with a PCA model guarantee an accurate estimation of internal skeletal points' location? In the present work, a surface matching error lower than $10 \mathrm{~mm}$ on average was obtained for all four tested methods. This is slightly higher than the mean error of reconstruction of the external envelope obtained from biplanar X-rays of $5 \mathrm{~mm}$ (Nérot et al., 2015b), suggesting that a good surface fit could be obtained using a reduced number of PCs from an existing sample datasets. Table 5 gives the Pearson correlation coefficients between the prediction errors of internal points in the anterior-posterior direction $(x)$ and those of external body surface as well as their correlations with different individual characteristics, such as body height, weight, age, T4-T12 kyphosis and L1-L5 lordosis angles. The prediction errors of all internal points, except for T1/T2 (whose prediction error was slightly correlated with surface matching error), were not correlated with the surface matching error, nor with a

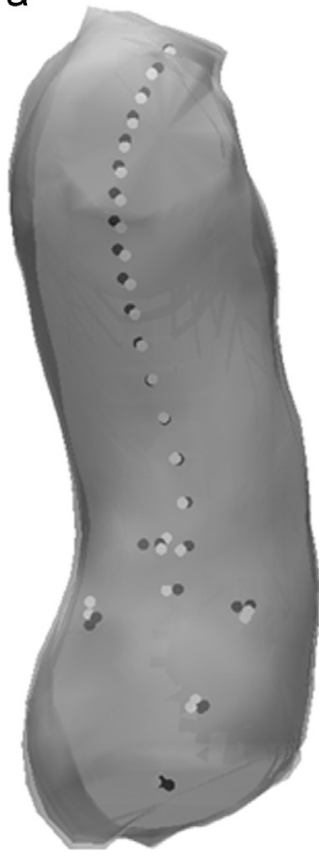

b

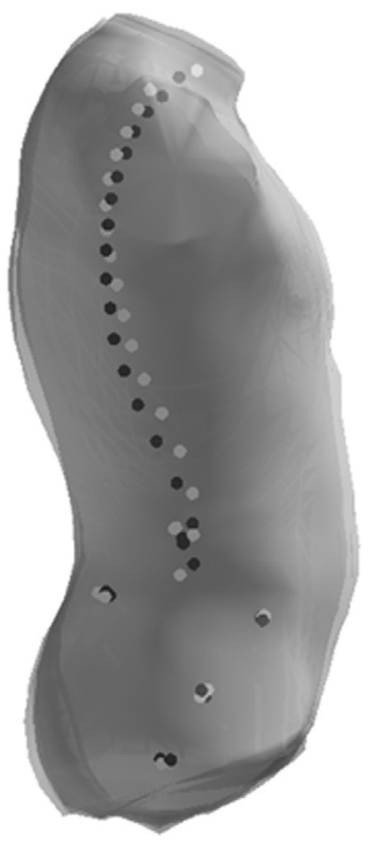

C

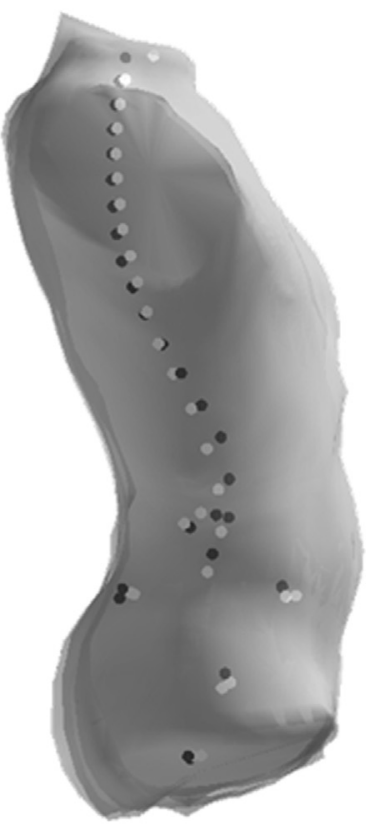

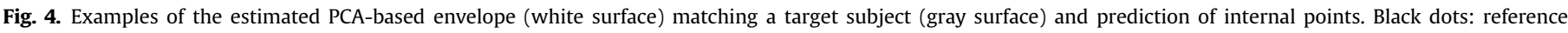

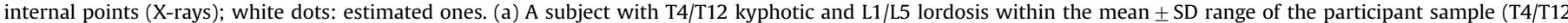
angle $34^{\circ}$, L1L5 lordosis angle: $34^{\circ}$ ) (b) a subject with a high T4T12 kyphotic angle of $54^{\circ}$ (c) a subject with a high L1L5 lordosis angle of $59^{\circ}$. 
weight, height or age. However a significant correlation was observed between the prediction error of thoracic joints and T4/T12 kyphosis angle, and between the prediction error of lumbar joints and L1/L5 lumbar lordosis angle. In particular, the prediction of L2/L3 was less accurate compared to other vertebral joints and its error of estimate was correlated with lumbar lordosis.

Fig. 4 compares three participants, one with T4/T12 kyphosis and L1/L5 lordosis angles close to the average values of the participants sample (mean \pm SD T4/T12 angle: $33.9^{\circ} \pm 11.0$; L1/L5 angle: $39.7^{\circ} \pm 8.2$ ) (4a), one with high T4-T12 kyphosis angle (4b) and one with high L1-L5 lordosis angle (4c). It was showed that kyphosis and lordosis angles were highly dependent on the pelvic incidence in the mechanism of maintenance of sagittal alignment (Legaye et al., 1998; Vaz et al., 2002; Skalli et al., 2007; Husson et al., 2010; Lazennec et al., 2013; Vialle et al., 2005). Pelvic incidence is a parameter defined as the angle between the perpendicular to the sacral plate at its midpoint and the line connecting this point to the femoral heads axis (Schwab et al., 2006). It cannot be easily estimated only from external body shape. Therefore even if a perfect estimation of the external envelope was obtained with the PCA model, an accurate prediction of internal points would not be guaranteed, as internal points' location also depends on other internal structures. This observation pointed out a limitation in the attempt of predicting internal points from external parameters. In the future, a way to improve spinal joint centers prediction from external body shape is to create PCA models based on a sample of the participants with very variable trunk curvature covering a large range of variation in lordosis and kyphosis angles. Other methods than PCA surface mapping could be considered with help a few external landmarks, such as C7 and T8 spinous processes along the dorsal midline in addition to the ASIS and PSIS. Work is in progress to investigate the relationship between the internal spine joint centers and external dorsal midline.

It should be noted that the proposed method requires acquisitions of the trunk 3D surface using surface scanning technologies which are not always standard lab equipment, despite the booming development of cheaper and smaller devices. We believe that such a 3D surface scanning device will be a part of motion analysis lab equipment in a near future especially when it is helpful in locating internal skeleton. Secondly the present study was limited to the analysis of a standing posture with accurate surface acquisition. However for many applications, postures of interest are quite different and the body shape could be hindered by object such as seat back. Future analyses should be performed to evaluate the applicability of the algorithm for the prediction of internal points from incomplete or less accurate shape model and from a strong deformed model in a seated position.

\section{Conclusion}

This study proposed and compared four different PCA-based methods for locating internal points from the external trunk envelopes. The average distance between the internal skeletal points as measured by the EOS and those predicted by the PCA mapping was less than $15 \mathrm{~mm}$ for all four proposed method, more accurate than skeletal models used in currently existing digital human models with one dimensional anthropometric dimensions as inputs. However, prediction errors of the thoracic and lumbar joints location was also found related to internal parameters that can be hardly predicted only from body surface. Work is in progress to identify the external predictors from the external body shape that are related to internal points' location. This should allow improving the prediction of internal points for the generation of external/internal subject-specific manikins.

\section{Conflict of interest}

The authors have no conflicts of interest to declare.

\section{Acknowledgments}

The clinical data used in this study were provided by the LBM/ Institut de Biomécanique Humaine Georges Charpak, Arts et Métiers ParisTech, Paris. The authors thank the ParisTech BiomecAM chair program on subject-specific musculoskeletal modeling, and in particular COVEA and Société Générale.

\section{References}

Allen, B., Curless, B., 2003. The Space of human body shapes: reconstruction and parameterization from range scans. ACM SIGGRAPH Comput. Graph., 27-31.

Anguelov, D., Srinivasan, P., Koller, D., Thrun, S., Rodgers, J., Davis, J., 2005. Scape: shape completion and animation of people. ACM Trans. Graph. 24, 408-416.

Aubert, B., Vergari, C., Ilharreborde, B., Courvoisier, A., Skalli, W., 2014. 3D reconstruction of rib cage geometry from biplanar radiographs using a statistical parametric model approach. Comput. Methods Biomech. Biomed. Eng. Imaging Vis., 1-15. http://dx.doi.org/10.1080/21681163.2014.913990.

Bell, A.L., Pedersen, R., 1989. Prediction of hip joint center location from external landmarks. Hum. Mov. Sci. 8, 3-16.

Bergeron, C., Cheriet, F., Ronsky, J., Zernicke, R., Labelle, H., 2005. Prediction of anterior scoliotic spinal curve from trunk surface using support vector regression. Eng. Appl. Artif. Intell. 18 (8), 973-983. http://dx.doi.org/10.1016/j. engappai.2005.03.006.

Blanchonnette, P., 2010. Jack Human Modelling Tool: A Review. Defense Science and Technology Organization, Australia.

Chaffin, D.B., 2005. Improving digital human modelling for proactive ergonomics in design. Ergonomics 48 (5), 478-491.

Chaibi, Y., Cresson, T., Aubert, B., Hausselle, J., Neyret, P., Hauger, O., de Guise, J.A., Skalli, W., 2012. Fast 3D reconstruction of the lower limb using a parametric model and statistical inferences and clinical measurements calculation from biplanar X-rays. Comput. Methods Biomech. Biomed. Eng. 15 (5), 457-466.

Croce, U. Della, Cappozzo, A., Kerrigan, D.C., 1999. Pelvis and lower limb anatomical landmark calibration precision and its propagation to bone geometry an joint angles. Med. Biol. Eng. Comput. 37 (2), 155-161.

Drerup, B., Hierholzer, E., 1996. Assessment of scoliotic deformity from back shape asymmetry using an improved mathematical model. Clin. Biomech. 11 (7), 376-383. http://dx.doi.org/10.1016/0268-0033(96)00025-3.

Dubousset, J., Charpak, G., Skalli, W., Deguise, J.A., Kalifa, G., 2010. Eos: a new imaging system with low dose radiation in standing position for spine and bone \& joint disorders. J. Musculoskelet. Res. 13 (01), 1-12. http://dx.doi.org/ 10.1142/S0218957710002430.

Faro, F., Marks, M.C., Pawelek, J., Newton, P.O., 2004. Evaluation of a functional position for lateral radiograph acquisition in adolescent idiopathic scoliosis. Spine 29, 2284-2289.

Harlick, J.C., Milosavljevic, S., Milburn, P.D., 2007. Palpation Identification of Spinous Processes in the Lumbar Spine. Man. Ther. 12 (1), 56-62. http://dx.doi.org/ 10.1016/j.math.2006.02.008 〈http://www.ncbi.nlm.nih.gov/pubmed/16781182〉.

Humbert, L., De Guise, J.A., Aubert, B., Godbout, B., Skalli, W., 2009. 3D reconstruction of the spine from biplanar X-rays using parametric models based on transversal and longitudinal inferences. Med. Eng. Phys. 31 (6), 681-687. http: //dx.doi.org/10.1016/j.medengphy.2009.01.003.

Husson, J.L., Mallet, J.F., Parent, H., Cavagna, R., Vital, J.M., Blamoutier, A., Violas, P., 2010. Applications in Spinal Imbalance. Orthop. Traumatol. Surg. Res. 96 (4), S1-S9. http://dx.doi.org/10.1016/j.otsr.2010.03.006.

Huysmans, T., Moens, P., Van Audekercke, R., 2005. An active shape model for the reconstruction of scoliotic deformities from back shape data. Clin. Biomech. 20 (8), 813-821.

Jolliffe, I.T. 2002. Principal component analysis, Second Edition. Encyclopedia of Statistics in Behavioral Science. Vol. 30 (3), p. 487. http://dx.doi.org/10.2307/ 1270093.

Lazennec, J.Y., Brusson, A., Rousseau, M.A., 2013. Lumbar-pelvic-femoral balance on sitting and standing lateral radiographs. Orthop. Traumatol. Surg. Res. 99, 87-103. http://dx.doi.org/10.1016/j.otsr.2012.12.003.

Legaye, J., Duval-Beaupère, G., Hecquet, J., Marty, C., 1998. Pelvic incidence; a fundamental pelvic parameter for three-dimensional regulation of spinal sagittal curves. Eur. Spine J. 7 (2), 99-103.

Nérot, A., Choisne, J., Amabile, C., Travert, C., Pillet, H., Wang, X., Skalli, W., 2015a. A 3D reconstruction method of the body envelope from biplanar X-rays: evaluation of its accuracy and reliability. J. Biomech. 48 (16), 4322-4326. http://dx. doi.org/10.1016/j.jbiomech.2015.10.044.

Mitton, D., Deschênes, S., Laporte, S., Godbout, B., Bertrand, S., de Guise, J.A., Skalli, W., 2006. 3D reconstruction of the pelvis from bi-planar radiography. Comput. Method Biomech. Biomed. Eng. 9 (1), 1-5. 
Nérot, A., Skalli, W., Wang, X., 2015b. An assessment of the realism of digital human manikins used for simulation in ergonomics. Ergonomics 58 (11), 1897-1909. http://dx.doi.org/10.1080/00140139.2015.1038306.

Park, B.K., Reed, M.P., 2014. Rapid generation of custom avatars using depth cameras. In: Proceedings of the 3rd International Digital Human Modeling Symposium. Tokyo, Japan.

Park, B.-K., Lumeng, J.C., Ebert, C.N., S.M, Reed, M.P., 2015. Child body shape measurement using depth cameras and a statistical body shape model. Ergonomics 58 (2), 301-309. http://dx.doi.org/10.1080/00140139.2014.965754.

Park, B.-K., Reed, M.P., 2015. Parametric body shape model of standing children ages 3 to 11 years. Ergonomics 58 (10), 1714-1725. http://dx.doi.org/10.1080/ 00140139.2015.1033480.

Peng, J., Panda, J., Van Sint Jan, S., Wang, X., 2015. Methods for determining hip and lumbosacral joint centers in a seated position from external anatomical landmarks. J. Biomech. 48, 396-400. http://dx.doi.org/10.1016/j.jbiomech.2014.11.040.

Quijano, S., Serrurier, A., Aubert, B., Laporte, S., Thoreux, P., Skalli, W., 2013. Threedimensional reconstruction of the lower limb from biplanar calibrated radiographs. Medical Engineering \& Physics 35, 1703-1712. http://dx.doi.org/10.1016/j.medengphy.2013.07.002 〈http://www.ncbi.nlm.nih.gov/pubmed/23938086〉.

Regazzoni, D., Rizzi, C., Colombo, G., 2015. The role of virtual ergonomic simulation to develop innovative human centered products. Digit. Hum. Model. Appl. Health Saf. Ergon. Risk Manag. Ergon. Health 9185, 74-83.

Reed, M.P., Raschke, U., Tirumali, R., Parkinson, M.B., 2014. Developing and implementing parametric human body shape models in ergonomics software. In: Proceedings of the 3rd International Digital Human Modeling Conference. Tokyo, Japan.

Reed, M.P., Manary, M.A., Schneider, L.W., 1999. Methods for measuring and representing automobile occupant posture. SAE Int. 108 (724), 1-14.
Reed, M.P., Park, B., Kim, K., Jones, M.L.H., 2015. Statistical prediction of body landmark locations on surface scans. In: Proceedings of the 19th thriennal congress of the IEA, Melbourne 9-14, 1 (August). pp. 1-4.

Schwab, F., Lafage, V., Boyce, R., Skalli, W., Farcy, J.P., 2006. Gravity line analysis in adult volunteers: age-related correlation with spinal parameters, pelvic parameters, and foot position. Spine 31 (25), E959-E967. http://dx.doi.org/10.1097/ 01.brs.0000248126.96737.0f.

Seidel, G.K., Marchinda, D.M., Dijkers, M., Soutas-Little, R.W., 1995. Hip joint center location from palpable bony landmarks: a cadaver study. J. Biomech. 28 (8), 995-998.

Sicard, C., Gagnon, M., 1993. A geometric model of the lumbar spine in the sagittal plane. Spine 18 (5), 646-658.

Skalli, W., Champain, S., Mosnier, T. 2007. Spine Biomechanics. Cahiers D'enseignement de La SOFCOT. Alternatives À L'arthodèse Lombaire. pp. 8-18.

Snyder, R., Chaffin, D.B., Schutz, R.K., 1972. Link System Of The Human Torso. Aerospace Medical Research Laboratory, Wright-Patterson Air Foderce base Ohio.

Vaz, G., Roussouly, P., Berthonnaud, E., Dimnet, J., 2002. Sagittal morphology and equilibrium of pelvis and spine. Eur. Spine J. 11 (1), 80-87. http://dx.doi.org/ $10.1007 / \mathrm{s} 005860000224$.

Vialle, R., Levassor, N., Rillardon, L., Templier, A., Skalli, W., Guigui, P., 2005. Radiographic analysis of the sagittal alignment and balance of the spine in asymptomatic subjects. J. Bone Jt. Surg. 87 (2), 260-267. http://dx.doi.org/ 10.2106/JBJS.D.02043.

Weiss, A., Hirshberg, D., Black, M.J. 2011. Home 3D body scans from noisy image and range data. In: Proceedings of the 2011 IEEE International Conference on Computer Vision (ICCV). pp. 1951-1958. 